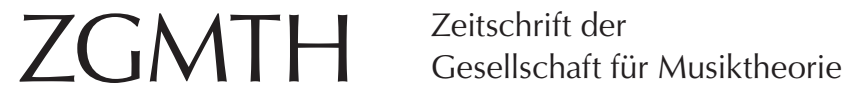

Nowak, Adolf (2017): Kritische Anmerkungen zu dem Aufsatz von Hermann Danuser: »Apollinische Fundamente. Über Adolf Nowaks Buch Musikalische Logik«, ZGMTH 13/2 (2016), 355-375. ZGMTH 14/2, 361-369. https://doi.org/10.31751/946

(C) 2017 Adolf Nowak (a.nowak@kunst.uni-frankfurt.de)

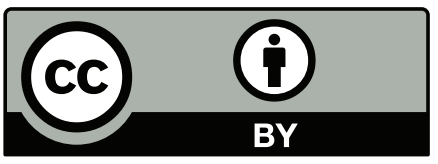

Dieser Text erscheint im Open Access und ist lizenziert unter einer Creative Commons Namensnennung 4.0 International Lizenz.

This is an open access article licensed under a Creative Commons Attribution 4.0 International License.

eingereicht / submitted: 02/10/2017

angenommen / accepted: 02/10/2017

veröffentlicht / first published: 31/12/2017

zuletzt geändert / last updated: 23/08/2018 


\title{
Kritische Anmerkungen zu dem Aufsatz von Hermann Danuser: »Apollinische Fundamente. Über Adolf Nowaks Buch Musikalische Logik«, ZGMTH 13/2 (2016), 355-375'
}

\author{
Adolf Nowak
}

\section{1. »Apollinisch«}

Die im Titel angekündigte These ist: Das Buch Musikalische Logik gebe »apollinische Fundamente« und wehre »alle Elemente einer sdionysischen`, im Zeichen einer Ästhetik des Erhabenen stehenden Kunst« ab (AF 369). Nicht gesagt wird, welche der im Buch besprochenen Werke - vom Alleluja Pascha nostrum mit seinen expansiven Melismen bis zur Cellosonate von Bernd Alois Zimmermann - als sapollinisch gelten sollen. Auch der Verfasser des Buchs weiß keine zu nennen, zumal ihn überzeugt, was Adorno zum vermeintlich Apollinischen bei Mozart gesagt hat. ${ }^{2}$ Grundsätzlich ist zu überlegen: Wenn es musikalische Logik gibt, dann kann sie nicht auf eine der ästhetischen Kategorien beschränkt werden, weder auf das 'Schönes im Sinne von Eduard Hanslick, noch auf das sApollinischer im Sinne von Friedrich Nietzsche. Musikalische Logik betrifft die Herstellung musikalischen Zusammenhangs, der sich als modellhaft und umwegfrei erweisen kann oder auch als Zusammenschluss dessen, was sich den gebahnten Wegen sperrt. Wenn musikalische Logik, dem Ansatz bei Augustinus entsprechend, das Auseinanderstrebende (distentio animi) trägt, dann wird sie weder das `Erhabene` ausblenden können noch das, was einer viel späteren Zeit `dionysisch heißt. Um 1870 spricht Nietzsche selbst - im Blick auf das Dionysische - von Logik! Das Dionysische, das in Ekstase, Rausch und Auflösung der Individuation gipfelt, hat seine Motivation in den »höchsten Lust- und Unlustzuständen des Willens « ${ }^{3}$. Der $>$ Wille im Sinne Schopenhauers ist ein Treiben widerstreitender Kräfte, er findet sein Abbild in konfliktiven Prozessen der Harmonik (concordia discors). ${ }^{4}$ Daran schließt Nietzsche an, wenn er - den Widerstreit als Zentrum der Logik anerkennend - in seiner Schrift "Die dionysische Weltanschauung»

1 Der Aufsatz »Apollinische Fundamente« wird im Folgenden unter AF, das Buch Musikalische Logik unter ML zitiert.

2 »Mozart jedenfalls, wenn man ihn schon apollinisch schimpfen will - wozu ich nicht das Herz hätte - steht in der Spannung ganz und gar: es bedarf keiner gar zu feinen Ohren, zu hören, wie grausam und schmerzlich diese Form aus dem Naturstoff geschnitten ward« (Stuckenschmidt/Adorno 1997, 451).

3 Nietzsche 1980a, 575.

4 Vgl. Schopenhauer 1949b, 514. 
sagt: »Der Wille und sein Symbol - die Harmonie - beide im letzten Grunde die reine Logik! «5 Fügt er dann hinzu, dass im Bereich des 'Willens` (und somit der Musik) der Begriff »ganz unmächtig" sei, so ist der Begriff als Sachbegriff gemeint (semantische Kodierung), während die »reine Logik« in Relationsbegriffen wirkt, aufgrund derer das Unbändige und das Geordnete, die Begrenzung und die Überschreitung, das Gefüge und die Auflösung zu erfahren sind. Es geht in musikalischer Logik um die Spannung zwischen solchen Gegensätzen respektive ihren technischen Korrelaten. Ohne Widerstand kann sich Logik gar nicht artikulieren. Die durch Logik entfalteten Ausdrucksweisen erreichen eine Differenzierung, die nicht auf eine einzelne ästhetische Kategorie, auch nicht auf eine Zweiheit wie die des Apollinischen und Dionysischen zu reduzieren ist. ${ }^{6}$

\section{Begriffsgeschichte und Problemgeschichte}

Der Entschluss zum binären Vergleich ${ }^{7}$ bringt Schematisierungen mit sich, die über manche Differenzen großzügig hinweggehen. Danuser ordnet das methodische Konzept des Buches der Begriffsgeschichte zu. Der entscheidende Ansatz ist aber die Überlegung, dass musiktheoretische und musikästhetische Texte nur dann verstanden werden können, wenn die Probleme rekonstruiert werden, auf die sie Antwort geben. Welche Probleme sind es, die die Musiktheorie und -ästhetik dazu brachten, Begriffe und Modelle der Logik zu bemühen? Diesen Ansatz - Theoriegeschichte, die die Rekonstruktion der Problematik anstrebt, auf welche die überlieferten Texte eine Antwort bieten - nannte ich sproblemgeschichtlich . Die Meinung, dass »das Problem« sich »beinahe zu verflüchtigen « scheint (AF 357), kann ich nicht nachvollziehen. Nicht von `dem ‘ Problem ist im Buch die Rede, sondern ausdrücklich von »verschiedenen, aus bestimmten geschichtlichen Situationen entstandenen Problemen [...], die die Theorie der Musik auf Logik, logische Begriffe und Muster verwiesen haben« (ML 5). Die geschichtlichen Problemlagen werden bereits in der Einleitung unter den Ziffern 1 bis 10 vorgestellt.

Danuser avisiert »deutliche Affinitäten« (AF 358) meines Buches zu seiner Dissertation Musikalische Prosa: in allgemeinen Vorgehensweisen wie Diachronie, Exemplifikation und Rekurs auf Ästhetik, speziell aber in der Bezugnahme auf Idee $\triangleleft .^{8}$ Nun kann unter >Idee`sehr Verschiedenes gedacht werden. Im Falle der musikalischen Prosa bezieht sich ıldee r auf Phänomene, die sich um die Metrik gruppieren (Rezitativ, Taktfreiheit, irreguläre Syntax und deren Folgen für Form und Stil). Die Idee musikalischer Logik dagegen meint zunächst keinen Phänomenbereich der Musik, sondern einen Anspruch, der an musikalische Phänomene gestellt wird. Die Idee ist hier ein Postulat, bei dem

5 Nietzsche 1980a, 574 (Hervorhebung original).

6 Vgl. in ML z.B. die Ausführungen zu Bruckner (260ff.), Berlioz (266f.), Reger (233ff.), Messiaen (316ff.).

7 Die erklärte Absicht ist, »Aspekte des Buchs Musikalische Logik im Vergleich mit eigenen Projekten zu beleuchten« (AF 355).

8 »Die stärkste Gemeinsamkeit indes liegt meines Erachtens im Umstand, dass auch Adolf Nowak, ohne dass wir uns jemals darüber ausgetauscht hätten, als leitendes Wort für seine Unternehmung ıIdees fixiert hat." (AF 358f.) 
nicht ausgemacht ist, ob es durch Harmonik, Metrik, Melodik, Rhythmik, Formbildung, Ausdruck oder erst im Zusammenwirken mehrerer oder aller Komponenten eingelöst werden kann. ${ }^{9}$ Wo die Idee nicht eine bestimmte Gegebenheit betrifft, sondern einen Anspruch, der an Phänomene nicht festgelegter Abgrenzung und Gewichtung gestellt wird, ist die Rekonstruktion der geschichtlichen Konstellationen gefordert, durch welche die jeweiligen Sachbereiche und der Anspruch an sie erst zu Tage treten. Daher handeln die Kapitel von so unterschiedlichen Phänomenen wie Zeitbewusstsein, Tonsystem, Melodiebildung, Generalbass, Priorität von Melodie oder Harmonie, thematischen Prozessen etc. Bei dieser Sachlage von `Idee` haben auch Exemplifikation und Rekurs auf Ästhetik eine andere Ausrichtung: Die Exemplifikation muss Tendenzen einbeziehen, die einem engeren Logik-Anspruch entgegenwirken (vgl. besonders die Kapitel: «Zur Logizität der modalen Komposition«; »Intention und Subintention in der Stimmführung«; »Aufhebungen der Kadenzlogik«). Der Rekurs richtet sich über die Ästhetik hinaus auf philosophische Logik.

\section{Begriff und Metapher}

Da die Begriffe ımusikalisches Denkenı, smusikalischer Gedanke und smusikalische Logikı in meinem Buch nicht als Außenbestimmungen der Musik, sondern als implizierte Bestimmungen aufgefasst werden, meint Danuser die «Abweisung eines metaphorischen Status der Begriffe« (AF 360) feststellen zu müssen (»dass sich der Autor, indem er eine metaphorische Lesart verwirft, dem Zwang eines Begriffsverständnisses aussetzt« [AF 364]). Tatsächlich halte ich die drei genannten Begriffsworte nicht für übertragene, sondern für phänomennahe Ausdrücke, aber zur Explikation dessen, was durch sie begriffen wird, können selbstverständlich und müssen sogar verschiedene Metaphern herangezogen werden. `Syllogismus`, `Gerichtsrede`(das Modell der rhetorischen Logik) und grammatische Begriffe z.B. werden als Metaphern eingebracht, um zu einem NichtMetaphorischen vorzustoßen: zu einer genuin musikalischen Logik, zu musikalischen Denkformen und Gedanken. Keineswegs möchte ich, wie Danuser offenbar annimmt, für Phänomene wie `Fortsetzung` und `Entwicklung` von Motiven den Terminus `Folgerung، einführen (AF 365). Wohl aber ist mir die Frage wichtig, ob es in den genannten Phänomenen Züge einer `Folgerung` gibt, die eine Analogie zum Syllogismus, nicht eine Gleichsetzung mit ihm, zulassen.

\section{Immanente Begriffe}

Gegen die Annahme einer der Musik immanenten Begrifflichkeit setzt Danuser das Insistieren »auf einem nicht-begrifflichen Fundament des Schaffens (als Komponieren, Improvisieren und Reproduzieren) «(AF 360), die Verwurzelung des Musikmachens »in begriffsloser Praxis" (AF 363). Begriffe sollen offenbar allein dem verbal formulierten Erkennen vorbehalten sein, sodass die Praxis der Musik nur insofern begriffsfähig wird,

9 Das Buch war zuerst unter dem Titel Musikalische Logik. Postulate - Modelle - Kontexte angekündigt. 
als sie, "wie andere Dimensionen des menschlichen Lebens, zu einem Gegenstand des Denkens, des Begreifens, der sprachlichen Verständigung gemacht wurde und bis heute wird.« (AF 363) Jegliche Praxis aber, sowohl der Musik als auch anderer Lebensbereiche, besteht in Handlungen, und Handlungen sind nicht ohne Entscheidungen möglich. Das Ent-scheiden wiederum setzt Unter-scheidungen voraus (dies zu wollen, auf diese Weise, jenes zu lassen oder sich dem Fluss momentanen Erlebens hinzugeben etc.). Solche Unterscheidungen brauchen nicht ausgesprochen zu werden, sie werden in der Entscheidungssituation im Sinne eines disjunktiven Urteils vollzogen und in diesem Vollzug werden sie leiblich begriffen, ohne oder auch mit Begleitung einer Reflexion, die das Begreifen verbalisiert. Entsprechend kann eine Tonfolge kompositorisch definiert werden: Durch die Weise ihres Gebrauchs zeigt sich, ob sie etwa als Wiederholung, als Variante, als Kontrapunkt etc. auftritt. Innerhalb der Aktion, nicht erst durch die Benennung, wird der funktionale Sinn der Tonfolge begriffen. ${ }^{10}$ Das Begreifen setzt in der Praxis an (Improvisieren, Hören), indem es Klänge zu Einheiten verschiedenen Grades verbindet. Der Begriff entspringt der »Handlung [des Verstandes], verschiedene Vorstellungen unter einer gemeinschaftlichen zu ordnen «11 (Kant). Der wissenschaftsgeschichtlich aktuelle Rahmen zu dieser Konzeption des Begriffs ist nicht, wie Danuser vorschlägt, die »Diskursgeschichte à la Foucault» (AF 360) - diese gilt dem verbalisierten Begriff -, sondern die erkenntnistheoretische Forschung zur Funktion des Begriffs in Wahrnehmung und Handlung und die spattern recognition der kognitiven Psychologie.

\section{Notwendigkeit und Freiheit, Konsequenz und Widerspruch}

Das Hegel-Zitat über Freiheit, deren Entscheidungsabfolge die Form von Notwendigkeit annimmt, versteht Danuser als erzwungene »Identität», die »heute in systematischer Sicht als passé« (AF 358) zu gelten habe. Es geht aber bei Hegel nicht um Identität, sondern um den Prozess eines Umschlagens in den Gegensatz. Wenn kompositorische Entscheidungen - in ihrer Sensibilität für die Ausdruckswerte des jeweiligen Materials und für Momente des Zufalls - nicht jederzeit willkürlich und widerrufbar sind, dann wird sich eine entscheidungsgenerierte Folgerichtigkeit einstellen. Dies ist die »Form von Notwendigkeit«, die Hegel meint und die nicht identisch ist mit einer mechanischen Notwendigkeit. Sie steht nicht den objektiv kausalen, sondern den subjektiv gesteuerten Vorgängen nahe, sie ist eine Notwendigkeit, die nicht gegeben, sondern herbeizuführen ist. Ihr entsprechen zweckgerichtete Handlungen in der Praxis ebenso wie die ıZweckmäßigkeit ohne Zweckı in der Poiesis. Es hätte nicht der Berufung auf Hegel bedurft, da die Unterscheidung von Freiheit und Willkür durch das Moment der Konsequenz einerseits viel ältere Wurzeln hat und anderseits auch in jüngerer, nicht an Hegel orientierter Philosophie ausgesprochen wurde, so z.B. bei Nietzsche, der das Kunstschaffen in

10 Vgl. in ML: »Begriff und Urteil innerhalb des musikalischen Denkens« (346-352), bes. 348-352.

11 »Alle Anschauungen, als sinnlich, beruhen auf Affektionen, die Begriffe also auf Funktionen. Ich verstehe aber unter Funktion die Einheit der Handlung, verschiedene Vorstellungen unter einer gemeinschaftlichen zu ordnen.«(Kritik der reinen Vernunft, A 68 / B 93; Kant 1975, 109f.) 
einem Bewusstsein von Notwendigkeit ansetzen lässt, das in einem Gefühl von Freiheit terminiert:

Die Künstler mögen hier schon eine feinere Witterung haben: sie, die nur zu gut wissen, dass gerade dann, wo sie Nichts mehr ıwillkürlich und Alles nothwendig machen, ihr Gefühl von Freiheit, Feinheit, Vollmacht, von schöpferischem Setzen, Verfügen, Gestalten auf seine Höhe kommt, - kurz, daß Nothwendigkeit und `Freiheit des Willens` dann bei ihnen eins sind..$^{2}$

Ein Grundgedanke meines Buches ist, dass musikalische Logik sich nicht nur in tradierter Typik und historisch bestimmbarer Konventionalität legitimiert (wie es in Ludwig Finschers Haydn-Monographie vorgeschlagen wird ${ }^{13}$ ), sondern dass sie auch die kompositorische Begründung der Modifikationen und Aufhebungen umfasst. Durch diesen Gedanken sieht sich Danuser veranlasst, an seinen Aufsatz »Das imprévu in der Symphonik» zu erinnern und gleichzeitig zu bezweifeln, dass »der Ausdruck `musikalische Logikı für das Gemeinte glücklich gewählt ist« (AF 366). Das imprévu ist ein Spezialfall, bei dessen Auftreten jeweils zu untersuchen ist, wie er sich zu der Konsequenz in den thematischen Prozessen verhält. Er kann also nicht eo ipso unter musikalische Logik subsumiert werden. Prinzipiell ist aber zu bedenken, dass musikalische Regeln sowohl als Grundlegung fungieren als auch einer Aufhebung anheimfallen können. Die Grundlegung provoziert Ausnahmen und die Aufhebung provoziert Neuregelung. Die Herausforderung des Gegensatzes darf von der Erforschung musikalischer Logik nicht ausgeschlossen werden. Daher waren für die historischen Konzepte musikalischer Logik jeweils drei Schritte zu gehen: a) das in Dokumenten der Musiktheorie als `logisch Benannte, die Formulierung des Postulats aufzusuchen; b) zu exemplifizieren, was dem als ^logisch Benannten entspricht bzw. es konkretisiert, begrenzt und modifiziert; c) das Nicht-Entsprechende, Entgegengesetzte heranzuziehen mit der Frage, ob es sich als unlogisch erweist oder eine Differenzierung des Begriffes musikalischer Logik herausfordert. Die Einbeziehung des Gegensatzes spielt eine große Rolle auch bei der Auseinandersetzung mit der Irreversibilität der Zeit (sodass dabei keineswegs, wie Danuser vermerkt, die Krebsstrukturen ausgeschlossen würden [AF 368, Anm. 30]) und bei der musikalischen Reproduktion, sofern diese aus nicht-identischen Perspektiven mit der Logizität des Werkes konfrontiert ist.

\section{Logik und verwandte Fachgebiete}

Logik steht in und seit der Tradition der artes liberales zwischen den sprachlichen und den mathematischen Wissenschaften. Nach beiden Seiten macht Danuser weitere Vorschläge. Einerseits hätte ich die mathematische Logik berücksichtigen, anderseits die Beziehungen zu Rhetorik und Topik verfolgen sollen (AF 364). Die Sache ließe sich noch weiter spannen: auf Seiten der mathematischen Logik zur Spieltheorie und zur Chaosfor-

12 Nietzsche 1980c, 148.

13 Finscher bezieht musikalische Logik auf »das Zusammenstimmen und die Folgerichtigkeit musikalischer Ereignisse in einem gegebenen formalen Rahmen und unter den Bedingungen historisch bestimmbarer Konventionen«(Finscher 2000, 147; vgl. dazu ML 252f.). 
schung, auf Seiten der sprachlichen Logik zur Transformationsgrammatik und zur Semiotik. Dass das weite Feld nicht als ganzes bearbeitet werden sollte, wurde im Nachwort deutlich ausgesprochen. Doch war nicht nur, wie Danuser vermutet, die »traditionelle» Logik heranzuziehen, welche er in den von Wilhelm Risse erforschten Logiktraditionen (16. bis 18. Jahrhundert) sieht (AF 364) ${ }^{14}$, sondern ebenso zu beachten waren ältere Logik-Konzepte einerseits sowie die transzendentale und die dialektische Logik anderseits. Was die neue Musik betrifft, so wurden mit Absicht nicht solche Komponisten befragt, die eine etablierte Art der Logik wie z.B. die eines mathematischen Verfahrens oder einer bestimmten Programmiersprache auf ihre Musik übertragen haben, sondern Komponisten, bei denen sich ohne solche Vorgaben (oder diesen entgegenarbeitend) eine Art von immanenter Logik (`Logizität der Zeitgestalt‘) ergibt.

\section{Quellen und Sekundärliteratur}

Die Unterscheidung sprimär und ssekundär scheint Danuser als Wertung aufzufassen, wenn er eine Musikologie fordert, »die auf keinem dienend-ssekundierenden verständnis fußt« (AF 363), und wenn er von »Primärautoren« spricht, hinter denen ein angeblicher »Sekundärautor« zurücksteht (AF 362). In den historischen Wissenschaften meint sprimärı die Dokumente historischer Positionen und ssekundärı nichts Geringeres als die wissenschaftliche Forschung zu den Dokumenten: sekundär also im Sinne von zweiter Reflexion (gegenüber der ersten, die im Dokument enthalten ist), nicht im Sinne von Zweitrangigkeit. Da Erwin Stein oder Josef Rufer aus einem Engagement für neue Musik im Sinne der Zweiten Wiener Schule schrieben, sind ihre Schriften Dokumente. Da Carl Dahlhaus in kritischer Reflexion die Forschung, auch und gerade anhand von Quellen der Musiktheorie, vorangetrieben hat, ist sein CEuvre gegenüber diesen Quellen Sekundärliteratur resp. Forschungsliteratur; diese kann ihrerseits zum Gegenstand der Forschung und somit zur Quelle werden. Angesichts der zahlreichen Literaturvorschläge und der Anmahnung einer Forschungsgeschichte zu einzelnen Autoren (AF 363) kann ich nur einbekennen, dass mir eine Beschränkung auf den engsten Kreis der jeweils behandelten Problematik dringend geboten scheint. Dass ich 'Satz und `Periode` ganz im Sinne der Schönberg-Schule zitiere, heißt nicht, dass sie mir "sakrosankte Pfeiler" der Formenlehre (AF 362) sind; vielmehr werden sie als historische Modelle für einfache logische Operationen eingesetzt: für das unausgesprochene hypothetische Urteil und für partielle Negation (ML 350f.). Warum das Kapitel über Heinrich Schenker nur als "Appendix« (AF 362) gelten soll und nicht als Auseinandersetzung mit einer der wichtigsten Quellen, ist mir nicht nachvollziehbar: Das Thema ist hier kein geringeres als die theoretische Aufhebung der Kadenzlogik. Von kompositorischen Aufhebungen handelt das darauf folgende Kapitel.

14 Das Verdienst Risses liegt allerdings gerade darin, die Vielgestaltigkeit der Positionen - gegen die übliche Vereinheitlichung zur straditionellen Logikı - aufzuzeigen. Der Titel des Werkes ist nicht »Die Geschichte der Logik« (AF 356), sondern Die Logik der Neuzeit (vgl. Risse 1964; 1970). 


\section{Zirkelverdacht}

Danuser sieht in meiner Argumentation »eine Gleichsetzung von 'Denken und eine petitio principii, wobei er auf die Seiten 12 und 343 verweist (AF 360f.). Auf Seite 12, in der Einleitung, wird betont, dass nicht nur die Logik - als Objektivation von Denkformen - in verschiedenen geschichtlichen Erscheinungsweisen aufgesucht werden muss, sondern auch das ımusikalische Denken`, das Hans Heinrich Eggebrecht den geschichtlichen Demarkationen musikalischer Logik als vermeintlich unbelastetes Potenzial entgegenstellen wollte. Auf Seite 343 wird zwischen psychologischen und logischen Zügen des Denkens unterschieden: Bewusstseinsstrom und Denkerlebnisse auf der einen Seite, logische Struktur der Argumentation auf der anderen. Von einer Gleichsetzung des Denkens mit Logik kann also nicht die Rede sein. Der Logik geht es um Denkformen und ihre Schlüssigkeit. Bei der Abgrenzung einer musikalischen Schlüssigkeit von der syllogistischen verwies ich auf eine musikalische Art der Folgerung (im Blick auf die motivische Entwicklung innerhalb eines Themas; siehe oben, Abschnitt 3). Auch hier wird mir ein Zirkelschluss unterstellt mit den Worten: »)Folgerung، setzt ein System ımusikalischer Logikı bereits voraus, kann es also nicht beweisen." (AF 365) Der Zirkelverdacht resultiert in diesem Fall aus einem quid pro quo: Zwei Intentionen werden verwechselt, das Aufsuchen und Vergleichen von Strukturen mit dem Beweisen eines Systems.

\section{Zu den »musikaffinen Philosophen«}

»Ist es denn ein Zufall«, fragt Danuser, »dass erwiesenermaßen musikaffine Philosophen - ich nenne nur drei: Arthur Schopenhauer, Friedrich Nietzsche und Ludwig Wittgenstein ,$-[\ldots]$ die, von eigener musikalischer Praxis erfüllt, sie in ihr philosophisches Denken aufnahmen, im Buch Musikalische Logik keinen Ort haben? (AF 369) Was diese Philosophen zum Thema Musik und Logik sagten, ist höchst bemerkenswert. In der Auswahl der Probleme allerdings, die im Buch verhandelt werden, schienen mir die möglichen Zitate nicht vordringlich. Schopenhauer nimmt Bezug auf die Streitfrage der Logik des Mittelalters über den Status der Allgemeinbegriffe (Universalien). Die Begriffe in verbaler Form sind die universalia post rem. Die Wirklichkeit der Welt enthält die universalia in re. Die Musik aber "giebt die universalia ante rem «"15, d.h. sie bringt die einander widerstreitenden und ergänzenden Kräfte des triebhaften 'Willens` zum Klingen, die dem Werden und Vergehen der Einzelexistenzen metaphysisch vorangehen. Es ist gewissermaßen das Negativ der theologischen Vorstellung, dass der ewige Geist wie ein Musiker handelt, der seinen Gedanken sinnlich wahrnehmbar machen will: »Agit enim mens aeterna quasi ut musicus, qui suum conceptum vult sensibilem facere. ${ }^{16}$ Nietzsche hat den Schopenhauer'schen Begriff des 'Willens` in seine Geburt der Tragödie übernommen, ausdrücklich auch die Beziehung der Musik auf die universalia ante rem ${ }^{17}$, die nicht in der mens aeterna als conceptum präexistieren, sondern energiegeladen als dissonant-

15 Schopenhauer 1949a, 311.

16 Nikolaus von Kues 1967, 524.

17 Vgl. Nietzsche 1980b, 106. 
harmonische Strebungen jenes `Willens` wirken. Eine Konsequenz dieser Deutung des Willens ist der oben in Abschnitt 1 zitierte Satz Nietzsches über den Willen und die konfligierende Harmonie: Beide seien, gegenüber der empirischen Wirklichkeit, »im letzten Grunde die reine Logik«, d.h. das Geflecht der Relationen, Muster und Triebkräfte, von denen die Wirklichkeit bestimmt ist. Neben der metaphysischen Verknüpfung von Musik und Logik gibt es bei Schopenhauer den empirischen Vergleich (die Logik verhalte sich zum vernünftigen Denken wie der Generalbass zur Musik $)^{18}$ und bei Nietzsche ein durch Carl Fuchs gewecktes Interesse an der Phrasierungslehre Hugo Riemanns ${ }^{19}$, die bekanntlich eine Spezifikation der auf Metrik bezogenen Logik ist. Zu Wittgenstein hat Katrin Eggers ein sehr lesenswertes Buch vorgelegt, in welchem die musikalische Logik eine bedeutsame Rolle spielt. ${ }^{20}$ An der Philosophie Wittgensteins sind auch die Forschungen orientiert, die von Ingolf Max an der Universität Leipzig (Institut für Philosophie, Abteilung Wissenschaftstheorie und Logik) über Logik der Harmonie durchgeführt werden. ${ }^{21}$

\section{Literatur}

Cadenbach, Rainer (2001), „Wie Hugo Riemann sich von Carl Fuchs dabei helfen ließ, >das erlösende Wort einmal bei Nietzsche zu finden. Zu einer vergessenen Kontroverse über künstlerisches Schaffen und `Phrasierung«", in: Hugo Riemann (18491919): Musikwissenschaftler mit Universalanspruch. Konferenzbericht Sondershausen 1999, hg. von Klaus Mehner und Tatjana Böhme-Mehner, Köln: Böhlau, 69-91.

Eggers, Katrin (2011), Ludwig Wittgenstein als Musikphilosoph, Freiburg i.Br.: Alber.

Finscher, Ludwig (2000), Joseph Haydn und seine Zeit, Laaber: Laaber.

Kant, Immanuel (1975), Kritik der reinen Vernunft [1781] (= Werke in zehn Bänden, Bde. 3-4), hg. von Wilhelm Weischedel, Darmstadt: Wissenschaftliche Buchgesellschaft.

Max, Ingolf (2017), »The Harmony of Colour Concepts: Bridging the Early and the Late Wittgenstein«, in: Colours in the development of Wittgenstein's Philosophy, hg. von Marcos Silva, Berlin: Springer, 339-371.

Nietzsche, Friedrich (1980a), „Die dionysische Weltanschauung« [1870], in: ders., Die Geburt der Tragödie. Unzeitgemäße Betrachtungen I-IV. Nachgelassene Schriften 1870-1873 (= Sämtliche Werke. Kritische Studienausgabe, Bd. 1), hg. von Giorgio Colli und Mazzino Montinari, München: de Gruyter, 551-577.

(1980b), Die Geburt der Tragödie aus dem Geiste der Musik [1872], in: ders., Die Geburt der Tragödie. Unzeitgemäße Betrachtungen I-IV. Nachgelassene Schriften 1870-1873 (= Sämtliche Werke. Kritische Studienausgabe, Bd. 1), hg. von Giorgio Colli und Mazzino Montinari, München: de Gruyter, 9-156.

18 Vgl. Schopenhauer 1949a, 53.

19 Vgl. Cadenbach 2001.

20 Vgl. Eggers 2011, bes. Teil I (27-73).

21 Vgl. Max 2017, bes. den Abschnitt »A Musical Intermezzo: The Logical Form of Chord Expressions and Their Operators« (353-358). 
_ (1980c), Jenseits von Gut und Böse [1886], in: ders., Jenseits von Gut und Böse; Zur Genealogie der Moral (= Sämtliche Werke. Kritische Studienausgabe, Bd. 5), hg. von Giorgio Colli und Mazzino Montinari, München: de Gruyter, 9-243.

Nikolaus von Kues (1967), Idiota de mente. Der Laie über den Geist [1450] in: ders., Philosophisch-theologische Schriften, lateinisch-deutsch, Bd. 3, hg. von Leo Gabriel, übersetzt von Dietlind und Wilhelm Dupré, Wien: Herder, 419-477.

Risse, Wilhelm (1964; 1970), Die Logik der Neuzeit (2 Bde.), Stuttgart-Bad Cannstatt: Frommann.

Schopenhauer, Arthur (1949a), Die Welt als Wille und Vorstellung, Bd. 1 [3. Auflage 1859] (= Sämtliche Werke, Bd. 2), hg. von Arthur Hübscher, Wiesbaden: Brockhaus.

(1949b), Die Welt als Wille und Vorstellung, Bd. 2 [3. Auflage 1859] (= Sämtliche Werke, Bd. 3), hg. von Arthur Hübscher, Wiesbaden: Brockhaus.

Stuckenschmidt, Hans Heinz / Theodor W. Adorno (1997), „Kontroverse über die Heiterkeit« [1930], in: Theodor W. Adorno, Musikalische Schriften VI (= Gesammelte Schriften, Bd. 19), hg. von Rolf Tiedemann, Frankfurt a.M.: Suhrkamp, 448-452. 